\title{
Hemorragia digestiva alta y divertículo duodenal. Reporte de un caso
}

\author{
Juan Jerónimo Solé, Hernán Figgini, Leonela Aloy, Otto Ritondale, Hugo Daniel Ruiz \\ Servicio de Cirugía General, Hospital Nacional Profesor Alejandro Posadas (Presidente Illia y Marconi). Morón, Buenos Aires, Argentina.
}

Acta Gastroenterol Latinoam 2020;50(2):169-172

Recibido: 14/10/2018 / Aceptado: 16/04/2019 / Publicado online: 29/06/2020

\section{Resumen}

El diverticulo duodenal sangrante es una patología infrecuente con necesidad de tratamiento de urgencia por su elevada mortalidad. Presentamos el caso clínico de un paciente de 72 años con múltiples comorbilidades, que ingresa con hemorragia digestiva alta, dolor abdominal e inestabilidad hemodinámica. Una vez lograda su estabilidad hemodinámica se realizó endoscopía digestiva alta, que evidenció divertículo duodenal en rodilla superior con sangrado en el fondo, por lo que se realizó control hemostático. TC: divertículo duodenal de base ancha a nivel de rodilla superior. Se programó cirugía por el riesgo de resangrado. En la cirugía se realizó diverticulectomía laparoscópica con sutura mecánica y se asoció colecistectomía. Informe anatomopatológico: pared duodenal con adelgazamiento parietal del duodeno, probable divertículo por pulsión. Se otorgó el alta con control radiológico al tercer día postoperatorio. El objetivo de este caso es mostrar la importancia de la resolución endoscópica de urgencia y el posterior manejo laparoscópico en un pa-

Correspondencia: Hugo Daniel Ruiz

Santos Dumont 2719, piso 8, dto. C. (C.P.: 1426). Ciudad Autónoma de Buenos Aires, Argentina

Correo electrónico: drhugoruiz@hotmail.com ciente con importantes factores de riesgo, que resultó en una corta estadía hospitalaria sin complicaciones postoperatorias.

Palabras claves. Divertículo duodenal, sangrado de tubo digestivo alto, dolor abdominal.

\section{Upper digestive hemorrhage and duodenal diverticulum. A case report}

\section{Summary}

Bleeding duodenal diverticulum is an infrequent pathology in need of emergency treatment due to its high mortality. We present the clinical case of a 72-year-old patient with multiple comorbidities, who was admitted with upper gastrointestinal bleeding, abdominal pain and hemodynamic instability. Once hemodynamic stability was achieved, upper digestive endoscopy was performed, showing duodenal diverticulum in the upper knee with bleeding in the fundus, performing hemostatic control. CT was requested: at the level of the upper duodenal knee, wide base diverticulum. Surgery was scheduled for the high risk of new bleeding. In surgery, cholecystectomy and diverticulectomy were performed laparoscopically with mechanical suture. Anatomopathological report: duodenal wall with parietal thinning of the duodenum, suggestive of diverticulum by pulsion. The discharge was granted with radiological control on the 3rd postoperative day. The aim of this case is to show the importance of endoscopic resolution of urgency and subsequent laparoscopic management in a patient with important risk factors, evidencing the short hospital stay without postoperative complications.

Key words. Duodenal diverticulum, upper digestive tract bleeding, abdominal pain. 


\section{Introducción}

El divertículo duodenal fue descrito por Chromel en 1710, y recién en 1951 Patterson y Bromberg publicaron el primer caso de hemorragia de tubo digestivo alto asociado a esta patología.

El duodeno es el segundo sitio más frecuente de los divertículos del tubo digestivo (el primero corresponde al colon), seguido por el yeyuno. La segunda porción del duodeno es el sitio más común (85-90\%). La tercera y cuarta porción representan el 10 y 20\%, respectivamente.

Son infrecuentes antes de los 30 años y su incidencia se incrementa con la edad, con un pico entre los 50 y 60 años; afecta más a las mujeres con una relación $\mathrm{H}: \mathrm{M} \mathrm{1:1,6.}$

Los divertículos duodenales pueden ser verdaderos (presentan todas las capas del intestino) o falsos o adquiridos (simples herniaciones de la mucosa y submucosa a través de la capa muscular). Los primeros suelen ser congénitos y menos frecuentes que los adquiridos o falsos. Estos últimos se localizan preferentemente en el borde mesentérico duodenal, como es el caso del presente reporte.

\section{Caso clínico}

Se presenta un paciente masculino de 72 años de edad, que ingresa con hemorragia digestiva alta con inestabilidad hemodinámica, EPOC severo, DBT tipo II e HTA en tratamiento con dos drogas.

Ingresa con hipotensión arterial, frecuencia cardíaca de $108 \mathrm{lpm}$ y frecuencia respiratoria de $24 \mathrm{rpm}$. Palidez mucocutánea generalizada, dolor abdominal a nivel de epigastrio acompañado de hematemesis y melena. Se realiza laboratorio que evidencia anemia (Hto 24\%, hemoglobina $6,4 \mathrm{mg} / \mathrm{l})$. Requirió transfusión de tres unidades de sangre y C-pap en unidad cerrada.

Evoluciona a las 24 horas hemodinámicamente estable. Se realiza endoscopía digestiva alta (VEDA) que informa: divertículo en rodilla duodenal superior con sangrado en el fondo del mismo. Se sospecha lesión de Dieulafoy, se realiza control hemostático con adrenalina y colocación de dos clips. Se realiza ecografía abdominal que muestra litiasis vesicular y dilatación duodenal, sin visualización del divertículo.

Se realiza TC (Figuras 1 y 2 ) que informa: en primera porción duodenal, a nivel de rodilla superior, divertículo de base ancha de 42 por $48 \mathrm{~mm}$ de diámetro, que luego de la administración de contraste se observa en toda su extensión.

El paciente pasa a sala general, presentando nuevo episodio de sangrado digestivo alto (melena) sin descompensación hemodinámica. Se realiza nuevamente
VEDA, que no muestra sangrado activo; se observa coágulo adherido en fondo de divertículo. VCC normal hasta ciego con hemorroides internas, resto sin particularidades.

Paciente estable, Hto 32\% y Hb 8,5 mg/l. Se externa con sulfato ferroso, complejo polivitamínico, bloqueantes $\mathrm{H} 1$, sucralfato y tratamiento de patologías de base con drogas habituales. Se programa colecistectomía y exploración laparoscópica del divertículo duodenal, por riesgo de resangrado.

En febrero del 2017 se realiza diverticulectomía con sutura mecánica translaparoscópica, con endograpadora de $3,5 \mathrm{~mm}$ de espesor, tutorizada con sonda de 36 french. Prueba de hermeticidad con azul de

Figura 1. TAC helicoidal, vista anterior donde se observa el divertículo.

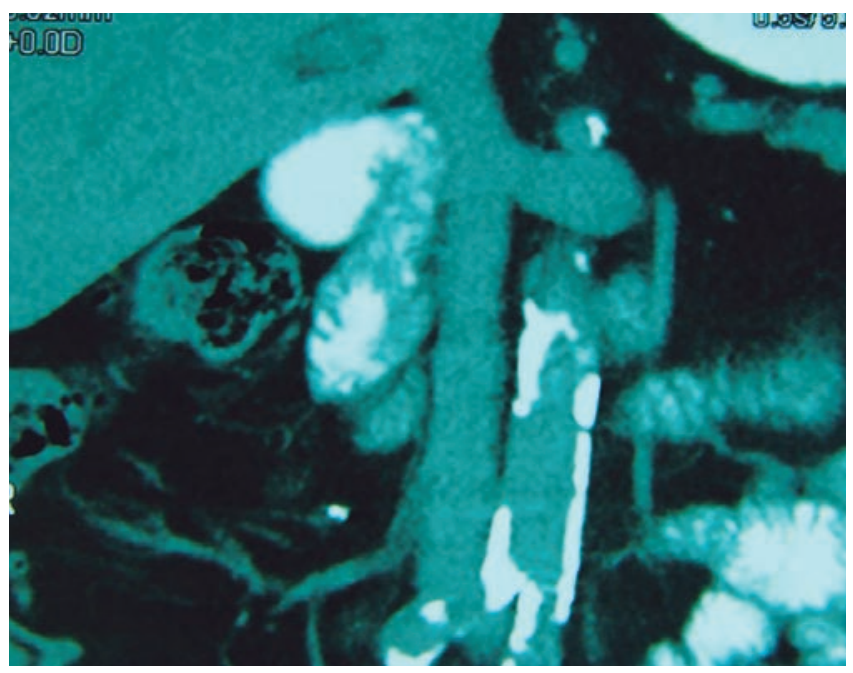

Figura 2. TAC helicoidal, corte coronal, donde se observa divertículo distendido y relación con otros órganos.

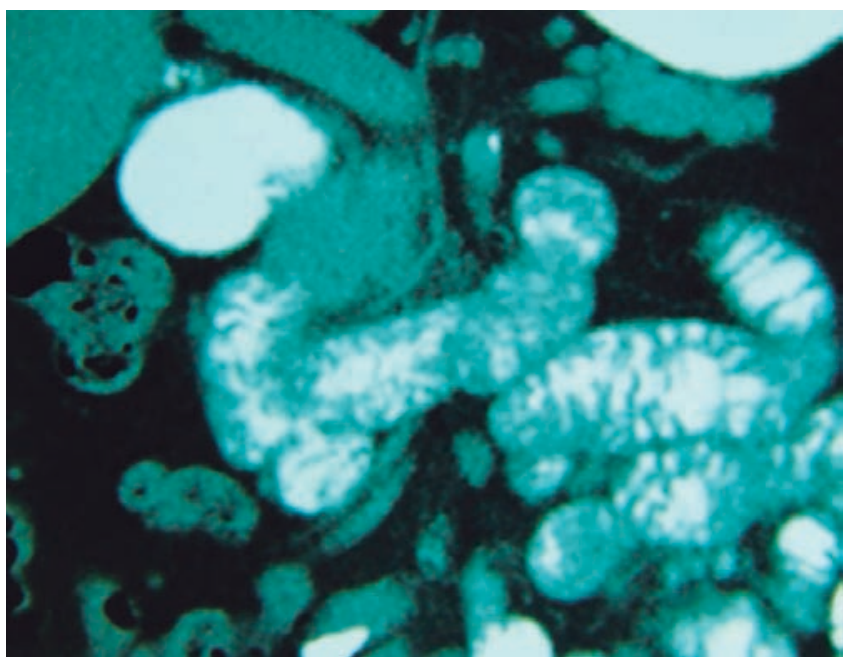


Metileno negativa, y se completa con colecistectomía laparoscópica. Anatomía patológica revela colecistitis crónica con macrolitos y pared duodenal con adelgazamiento parietal de duodeno, probable divertículo por pulsión (Figura 3).

El paciente presenta buena evolución postoperatoria, se realiza SEG control (Figura 4) postquirúrgico previo al alta.

\section{Figura 3. Vista postoperatoria de divertículo y vesícula biliar} extirpada.

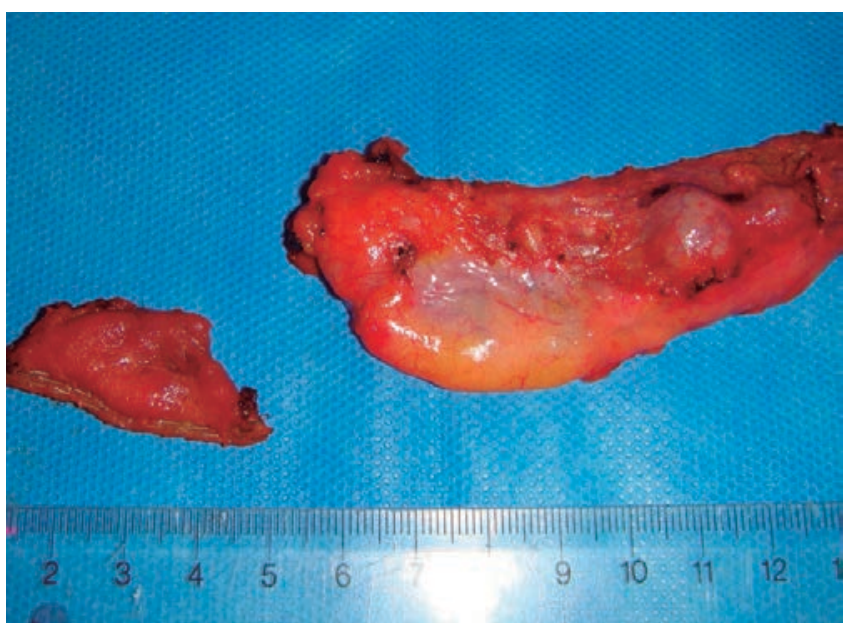

Figura 4. Seriada esofagogastroduodenal, SEG control posquirúrgico. Se observa buen pasaje de contraste.

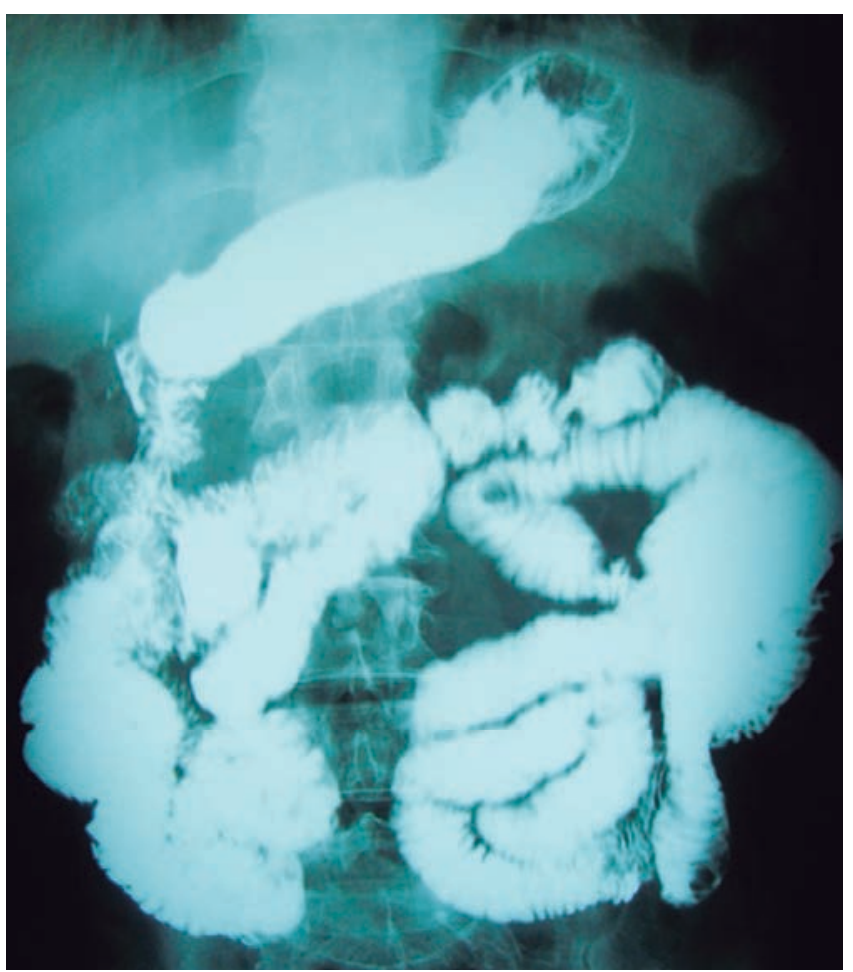

Se otorga egreso sanatorial al tercer día de la cirugía, con dieta líquida con proteínas. No presentó complicaciones postoperatorias.

\section{Discusión}

La enfermedad diverticular duodenal es asintomática en el $90 \%$ de los casos. Las complicaciones son raras pero la morbimortalidad es significativa. Su incidencia se desconoce, está presente en $0,16 \%$ a $5 \%$ de la población en estudios gastrointestinales contrastados y en la colangiografía endoscópica entre el 9 y el 23\%., ${ }^{2,3}$

La hemorragia de tubo digestivo alto es un motivo importante de consulta en la urgencia. La mayoría de las entidades que producen hemorragia digestiva requieren manejo inmediato debido a la posibilidad de inestabilidad hemodinámica e incremento en el riesgo de morbimortalidad. ${ }^{4}$

Las hemorragias de divertículos duodenales se originan principalmente por páncreas divisum, ectopia pancreática y también la lesión de Dieulafoy. ${ }^{5}$ Esta última es más frecuente en varones de edad avanzada con comorbilidades cardiorrespiratorias. Nuestro paciente reúne dichas características, pero no se confirmó por anatomía patológica.

El diagnóstico del divertículo duodenal sintomático es complejo y a menudo erróneo debido a que el paciente presenta semiología inespecífica que obliga a descartar otras etiologías mediante endoscopía alta, seriada esofagogastroduodenal con bario, tomografía con doble contraste o hidrotomografia, arteriografía selectiva o como hallazgo intraoperatorio. ${ }^{6-8}$ En este caso, la endoscopía digestiva alta permitió el diagnóstico y el tratamiento de urgencia, mientras que la tomografía con contraste oral y endovenoso evidenció la patología y permitió la diagramación del abordaje quirúrgico.

El tratamiento definitivo con menor morbimortalidad de esta patología es la diverticulectomía con sutura mecánica o manual por vía laparoscópica, especialmente en pacientes con comorbilidades. ${ }^{8}$

\section{Conclusión}

Los divertículos duodenales sangrantes son una patología muy infrecuente, la endoscopía digestiva y la tomografía con contraste oral y endovenoso confirman el diagnóstico. La mejor resolución para la hemorragia digestiva es la endoscopía; si no es efectiva debe tratarse con hemostasia directa laparoscópica o laparotómica. La cirugía electiva fue segura, incluso se asoció a colecistectomía laparoscópica por litiasis vesicular sintomática, sin complicaciones, permitiendo el egreso hospitalario al tercer día. 


\section{Conflicto de intereses. No existen conflictos de interés.}

\section{Referencias}

1. Pérez LR, Martínez MM, Rened FP. Hemorragia digestiva alta masiva secundaria a lesión de Dieulafoy oculta en un divertículo duodenal. Rev Esp Enferm Dig 2017; 109: 876-877.

2. González JA, Zaizar-Magaña A. Divertículo duodenal en la tercera porción del duodeno como causa de sangrado del tubo digestivo alto y dolor crónico abdominal. Informe de un caso y revisión de la literatura. Cir Ciruj 2008; 76: 65-69.

3. Anand V, Provost J, Bakr M, Bach C, Merchant P, Brown C, Gruss C. Two Cases of Intraluminal "Windsock" Diverticula Resultingin Partial Duodenal Obstruction. ACG Case Rep J 2016; $3(4): 135$.
4. Valdivieso Cortázar E, Alonso M, Marín S, Wörner P, Aguirre P. Hemorragia diverticular duodenal: un reto endoscópico. Rev Esp Enferm Dig 2017; 109: 468-469.

5. Sanz M, Román M, Yuste R. Lesión de Dieulafoy en divertículo duodenal. Una causa infrecuente de HDA. Rev Esp Enferm Dig 2018; 110: 266-267.

6. Zárate J, Rovelo J, Castillo V, Vivanco D, Espeje Ll. Divertículo duodenal como causa de sangrado de tubo digestivo. Informe de un caso y revisión de la literatura. Rev Invest Med Sur Mex 2010; 17: 18-20.

7. León C, Lucena JL, Chaparro M, Jiménez E, Pueyo A, Giménez L, Román L. Manejo quirúrgico del divertículo duodenal causante de hemorragia digestiva alta. Cir Esp 2017; 95: 346.

8. Dilip D, Nigel V, Maharaj R, Hariharan S, Vijay N. Laparascopy diverticulectomy for massive hemorrhage in a duodenal diverticulum. Surg Laparosc. Endosc. Percutan Tech. 2012; 22: 39-41. 